\title{
THE INFLUENCE OF CHEMICAL COMPOSITION AND HEAT TREATMENT OF STEEL FORGINGS ON MACHIN. ABILITY WITH SHALLOW LATHE CUTS
}

\author{
By T. G. Digges
}

\section{AESTRACT}

The tests described in this report were made primarily as a study of lathe-tool performance with shallow cuts as affected by variations in chemical composition and heat treatment of the steels cut. The cutting tests were made dry with high-speed steel tools of a selected size, form, composition, and heat treatment, with a feed of 0.0115 inch per revolution and 0.010 inch depth of cut. Comparisons were made of the Taylor speeds on the basis of equal tensile strengths when cutting 0.4 per cent carbon (S. A. E. 1040), chromium-vanadium (S. A. E. 6140), nickel-chromium (S. A. E. 3140 and 3435), chromium-molybdenum (S. A. E: 4140), and 31/2 per cent nickel (S. A. E. 2340) steel forgings heat treated to give tensile strengths between 75,000 and 220,000 lbs./in. ${ }^{2}$

This study also included consideration of the surface finishes of the various steel forgings as affected by the test conditions, the microstructures of the steels cut, and tool performance as affected by the additions of 3.5 to 11.7 per cent cobalt to the customary 18 per cent tungsten type of high-speed tool steel.

If machinability is measured by the cutting speed permitting the tools to last a definite time, then measurable differences were observed between the various steels cut in the lathe test with shallow cuts. The fact, however, that some given steel permits a higher cutting speed than another steel for some tensile strength which is the same for both materials does not necessarily indicate that the two steels maintain the same relationship for another tensile strength.

Of the different steels cut in the lathe tests the plain carbon steel was the most difficult to machine other than an annealed nickel-chromium steel. The surface finish on the plain carbon steel was also considered to be inferior to that of the alloy steels.

The results showed that the effect of changes in chemical composition of steel forgings upon their cutting speeds was dependent upon the tensile strength at which the comparisons were made. In the different steels cut with shallow cuts the most effective special alloying elements for improving machinability were the combinations of nickel and chromium or chromium and vanadium for the high tensile strengths in the neighborhood of $180,000 \mathrm{lbs} . /$ in. $^{2}$, while chromium and molybdenum were the most effective in the lower range of about $90,000 \mathrm{lbs} . /$ in. $^{2}$

\section{CONTENTS}

I. Introduction

III: Method of test

IV: Results of cutting tests

V: Tests with cobalt high-speed steel tools _...................... $98 \mathrm{~S}$

VI. Microstructures and surface finishes. . . _...

VII. Summary and conclusions .

VIII. Acknowledgments. .

\section{INTRODUCTION}

During the past 10 years a study has been made at the National Bureau of Standards of lathe-tool performance with both roughing and shallow cuts. The lathe breakdown test for roughing cuts was 
used in studying the effects upon tool performance of changes in chemical composition and heat treatment of commercial and experimental high-speed steels ${ }^{123}$ and of variations in chemical composition and heat treatment of the steels cut. ${ }^{4}$ The lathe breakdown test has also been extended to include cutting tests with cemented tungsten carbide tools. ${ }^{5}$

A method for testing lathe tools with shallow cuts was developed and used for studying the effects upon tool performance of changes in chemical composition and heat treatment of the tools. Relations hetween the cutting speed, feed, and depth of cut and the tool life for carbon and high-speed steel tools were also determined. ${ }^{6}{ }^{7}$ Most of the tests with shallow cuts were made in cutting $3 \frac{1}{2}$ per cent nickel steel forgings, heat treated to have tensile strengths of about 80,000 to $100,000 \mathrm{lbs} . /$ in. $^{2}$

The tests described in this report were made primarily as a study of high-speed steel lathe-tool performance with shallow cuts as affected by variations in the chemical composition and heat treatment of the steels cut. The study also included consideration of the surface finishes of the various steel forgings as affected by the conditions of cutting and of the microstructures of the steels cut.

The lathe tests were made in cutting a plain carbon and various alloy steel forgings heat treated to have tensile strengths between 75,000 and $220,000 \mathrm{lbs} . / \mathrm{in}^{2}{ }^{2}$ The form, size, heat treatment, and composition of the high-speed steel tools were not varied except that a study was made of tool performance as affected by the addition of cobalt to the 18 per cent tungsten type of tool steel.

In most cases the limiting factor of machinability is tool failure, and machinability can best be measured in terms of the cutting speed permitting a definite tool life; that is, machinability is proportional to the cutting speed permitting a definite tool life, and it is upon this basis that the term is used in this report. From this it follows that those materials which under otherwise fixed conditions permit the longest cuts without regrinding of the tools are described as the most readily machinable or to have the highest degree of machinability.

With finishing cuts, close adherence to dimensions and the nature of the finish left on the work piece are probably as important factors as tool life. However, no very satisfactory method has yet been worked out for the evaluation of the appearance or type of finish produced by a cutting tool. ${ }^{8}$ The method of test selected made possible a close adherence to the desired dimensions.

I H. J. French and Jerome Strauss, Lathe Breakdown Tests of Some Modern High-Speed Tool Steels, B. 8. Tech. Paper No. 228, also Trans. A. S. S. T., 2, D. 1125; 1922.

U. J. French, Jerome Strauss, and T. G. Digges, Effect of Heat Treatment on Lathe Tool Performance nnd Eome Other Properties of High-Speed Steels, Trans. A. S. S. T., 4, p. 353; 1923.

'II. J. French and T. G. Digges, Experiments with Nickel, Tantalum, Cobalt, and Molybdenum in High-Speed Steels, Trans. A. S. S. T , 8, . 681; 1925

- II. French and T. G. Digges, Rough Turning with Particular Reference to the Steel Cut, Trans. A. S. M. E., 45, P. 533; 1926 .

T. G. Digges, Cutting Test with Cemented Tungsten Carbide Lathe Tools, B. S. Jour. Researeh (12 PaOB), s, P. 365; 1930; also Trans. A. S. M. E. NISP-52-13, p. 155

- II. J. French and T. G. Digses, Effects of Antimony, Arsenic, Copper, and Tin in High-Speed Tool Steel, Trans. A. S. S. T., 13, p. 919; 1923.

i I1. J. French and T. G. Digges, Turning with Shallow Cuts at High Speeds, B. S. Jour. Research (RP120), 8, p. 820; also Trans. A. S. M. E. MISP-52-6, p. 55.

IR. F. W. Harrison, A Survey of Surface Quality Standards and Tolerance Based on 1929-30 Precision AHnding Practice. Paper presented at annul meeting A.S. M. I., 1930. The finish calibrator deseribed In this paper could possibly have been adapted to the work described in the present report and might have kiven results of more value than did the method used for evaluation of the surface finish. However, the wherimental work of the present investigation was practleally completed before the report by Harrison 


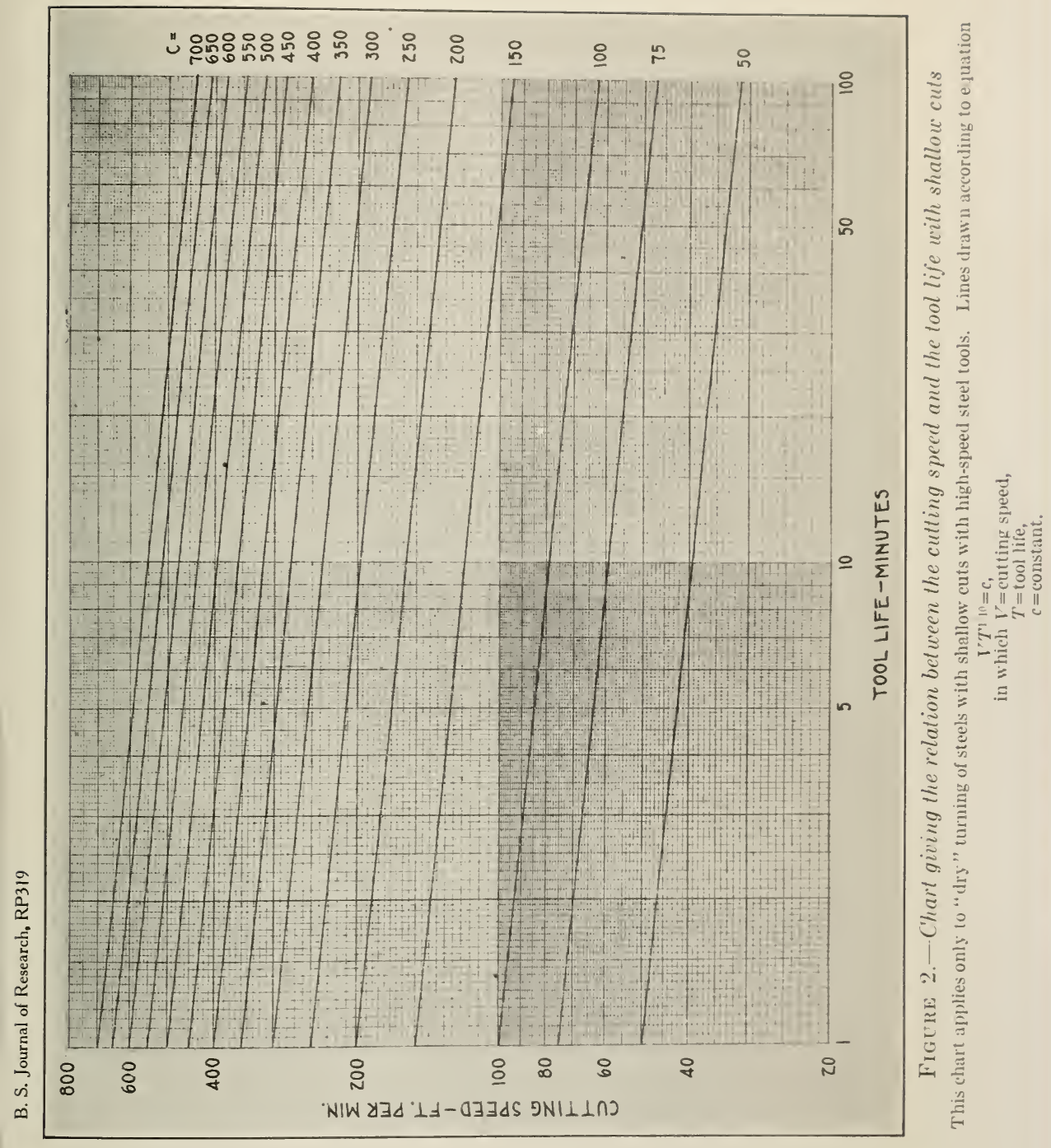




\section{PREVIOUS INVESTIGATIONS}

The results of previous lathe tests ${ }^{9}$ made with high-speed stecl tools with shallow cuts showed that with a constant feed and depth of cut there was a continuous increase in tool life as the cutting speed was decreased which could be represented approximately by the
equation

in which

$$
V T^{n}=V_{\mathrm{o}} T_{\mathrm{o}}{ }^{n}=c
$$

$V=$ cutting speed, feet per minute.

$T=$ tool life, minutes.

$T_{\mathrm{o}}=20$ minutes.

$V_{\mathrm{o}}=$ Taylor speed; that is, the speed which would give a tool life of 20 minutes.

$c=a$ constant for the metal (with specified heat treatment) which is being cut.

$n=$ a constant experimentally determined by varying $V$.

$A$

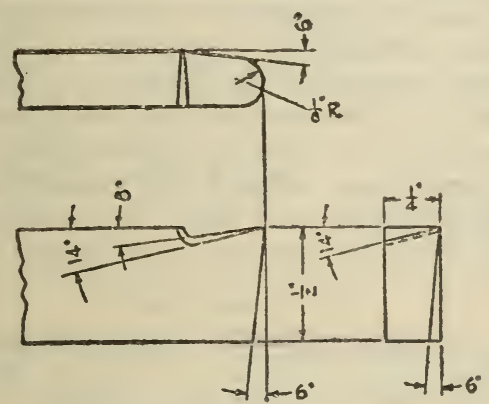

B

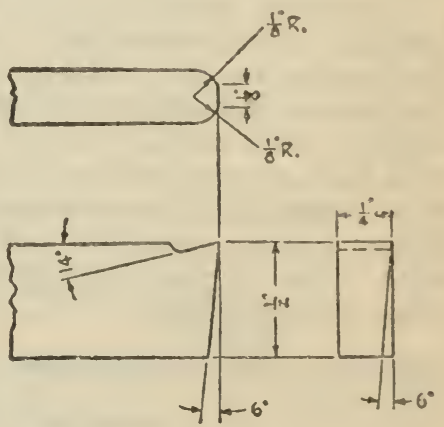

Figtre 1.-Dimensions and forms of the tonls used in the lathe tests $A$, tool for roughing cuts; $B$, tool for shallow cuts.

The tests made at different cutting spceds with a feed of 0.015 inch per revolution and depths of cut of 0.010 and 0.020 inch showed that within a range of tool life of from 2 to 125 minutes, the experimental results could be closely represented by equation (1) with $n=1 / 10$. This equation with $n=1 / 10$ has therefore been used in the present report for all computations involving the relation between the cutting speed and tool life and gives results sufficiently accurate for all practical purposes except when extrapolating for very long tool lifo from tests of short duration.

Equation (1) with $n=1 / 10$ was established with broadnose highspeed steel tools, illustrated in Figure $1(B)$, in dry cutting $3 \frac{1}{2} \mathrm{per}$ cent nickel steel forgings having tensile strengths between 80,000 to $100,000 \mathrm{lbs}$./in. ${ }^{2}$. This equation is represented graphically by straight lines when log-arithmetic coordinates are used. The chart shown in Figure 2 for finishing cuts is of a convenient form for computing the life of tools at various speeds. Numerically the constant " $c$ " is the cutting speed corresponding to a tool life of one minute.

- See footnote 7, p. 978.

$55946^{\circ}-31-5$ 


\section{METHOD OF TEST}

The method used for testing lathe tools with shallow cuts has heen described in detail in a previous report. ${ }^{10}$ With this method the test and indicating tools were set at equal depths in a special tool holder at the start of the test. The indicating tool began to cut when the wear on the test tool was from 0.001 to 0.002 inch, and this was considered as the point of failure of the test tool. In most cases it was found that the wear of 0.001 to 0.002 inch coincided with a complete breakdown of the tool comparable to that found with heavy cuts in rough turning.

From 6 to 10 tools were tested for each condition investigated and only arerage values of tool life were used for computation and comparison purposes.

The lathe tests with shallow cuts were made with high-sped steel tools of the broad-nose type, having dimensions and form shown in Figure $1(B)$. A round nose tool (fig. $1(A)$ ) was used for the indicator. Chemical composition and heat treatment of the high-speed steels used are given in Table 1.

The pieces on which the cuts were made, commonly referred to as forgings, were selected with the view of obtaining representative steels that come to the ordinary shop for machining operations. The chemical compositions of the forgings are given in Table 2. The forgings were initially about 8 inches in diameter and hollow bored with holes approximately 3 inches in diameter. Some of the forgings, after being used for a series of cutting tests, were heat treated to higher tensile strengths and hardness values and used for further tests. The forgings having the same S. A. E. number were from the same original forging.

TABLE 1.-Chemical composition and heat treatment of the lools used in the lathe tests

\begin{tabular}{|c|c|c|c|c|c|c|c|c|c|c|c|c|}
\hline \multirow{2}{*}{$\begin{array}{l}\text { Eterl } \\
\text { No. }\end{array}$} & \multicolumn{10}{|c|}{ Chemical composition } & \multirow{2}{*}{$\begin{array}{l}\text { Quenched } \\
\text { from }^{1}\end{array}$} & \multirow{2}{*}{$\begin{array}{l}\text { Temperer } \\
\text { at ? }\end{array}$} \\
\hline & C & Mn & $\mathrm{P}$ & S & $\mathrm{Si}$ & $\mathrm{Cr}$ & w & $\mathrm{V}$ & $\mathrm{Co}$ & Mo & & \\
\hline $\begin{array}{l}\mathrm{A} A \ldots \\
\mathrm{B} B \ldots \\
\mathrm{B} \text { С } \ldots . . .\end{array}$ & $\begin{array}{c}\text { Per dt. } \\
0.68 \\
.70 \\
.68 \\
.79\end{array}$ & $\begin{array}{c}\text { Per d. } \\
0.20 \\
.30 \\
.17 \\
.42\end{array}$ & $\begin{array}{c}\text { Per ct. } \\
0.031 \\
.030 \\
.030\end{array}$ & $\begin{array}{l}\text { Per ct. } \\
0.013 \\
.031 \\
.005\end{array}$ & $\begin{array}{c}\text { Per ct. } \\
0.21 \\
.14 \\
.34 \\
.15\end{array}$ & $\begin{array}{c}\text { Per ct. } \\
3.79 \\
3.68 \\
4.23 \\
4.57\end{array}$ & $\begin{array}{r}\text { Pcr ct. } \\
18.2 \\
17.6 \\
18.3 \\
21.5\end{array}$ & $\begin{array}{c}\text { Per ct. } \\
0.99 \\
1.18 \\
1.53 \\
1.47\end{array}$ & $\begin{array}{r}\text { Per ct. } \\
4.5 \\
7.8 \\
11.7\end{array}$ & $\begin{array}{r}\text { Per ct. } \\
0.05 \\
.96 \\
.61\end{array}$ & $\begin{array}{l}{ }^{\circ} F . \\
2,400 \\
2,450 \\
2,450 \\
2,450\end{array}$ & $\begin{array}{r}{ }^{\circ} F_{1} \\
1,100 \\
1,100 \\
1,100 \\
1,100\end{array}$ \\
\hline
\end{tabular}

1.1] tools were first annealed by heating for 2 to 3 hours at 1,600 to $1,650^{\circ} \mathrm{F}$. and furnace cooled. They were preheated for 20 minutes at $1,600^{\circ} \mathrm{F}$, and then held $11 / 2$ minutes in the high-temperature furnace at the tempersture Indicated.

' Tools were heated in the furnace to the temperature indicated, held 30 minutes, and air cooled.

TABLE 2.-Chemical composition of the forgings cut in the lathe tests

\begin{tabular}{|c|c|c|c|c|c|c|c|c|c|c|}
\hline \multirow{2}{*}{$\begin{array}{l}\text { Forg. } \\
\text { Ing } \\
\text { Nio. }\end{array}$} & \multirow{2}{*}{$\begin{array}{l}\text { S. A. E. } \\
\text { No. }\end{array}$} & \multicolumn{9}{|c|}{ Chemical composition a } \\
\hline & & C & $M n$ & $P$ & $\mathrm{~S}$ & Si & $\mathrm{Cr}$ & V & $\mathrm{Ni}$ & Mo \\
\hline $\begin{array}{l}46 \\
17 \\
45 \\
40 \\
80 \\
31\end{array}$ & $\begin{array}{l}6140 \\
4140 \\
3435 \\
3140 \\
2340 \\
1040\end{array}$ & $\begin{array}{c}\text { Per cent } \\
0.39 \\
.39 \\
.39 \\
.37 \\
.31 \\
.42\end{array}$ & $\begin{array}{c}\text { Per cent } \\
0.71 \\
.5 B \\
.59 \\
.64 \\
.73 \\
.65\end{array}$ & $\begin{array}{c}\text { Per cent } \\
0.019 \\
.020 \\
.017 \\
.021 \\
.026 \\
.020\end{array}$ & $\begin{array}{c}\text { Per cent } \\
0.029 \\
.023 \\
.019 \\
.024 \\
.025 \\
.015\end{array}$ & $\begin{array}{c}\text { Per cent } \\
0.20 \\
.22 \\
.26 \\
.20 \\
.20 \\
.19\end{array}$ & \begin{tabular}{c} 
Per cent \\
0.95 \\
.86 \\
.76 \\
.50 \\
- \\
\hdashline
\end{tabular} & $\begin{array}{c}\text { Per cent } \\
0.17\end{array}$ & \begin{tabular}{c} 
Per cent \\
2.96 \\
1.26 \\
3.43 \\
\hdashline
\end{tabular} & $\begin{array}{c}\text { Per cent } \\
0.17 \\
\end{array}$ \\
\hline
\end{tabular}

- Chemleal analysis data reported by the manufacturers.

"fee inntnote 7, P. פr8. 
The heat treatments and corresponding average mechanical properties of the forgings are given in Table 3. Average mechanical properties were determined from two or more specimens cut longitudinally from the forgings so as to represent the average properties of the metal removed during cutting. Likewise, the micrographs were made on sections representing the metal removed during the cutting tests.

The hardness of some of the forgings after heat treatment was above the range of what is considered commercially machinable with the present high-speed steel tools. This condition macie possible a survey of the entire machinable range from the solt or annealed steel to the upper limit of the usual machinability range.

All cutting tests were made "dry" with a constant feed of 0.0115 inch per revolution, depth of eut of 0.010 inch and variable speed, depending upon the properties of the metal cut. The cutting speed was varied with the different steel forgings, but was kept constant for each series of tests on a giren forging with given mechanical properties. In general, the cutting speeds selected were such as to give a tool life of from 10 to 30 minutes as is shown in Table 4 . From the data thus obtained the cutting speed permitting a 20-minute tool life (Taylor speed) was computed from the average tool-life values by means of equation 1 with $n=1 / 10$ or by extrapolating by using the chart shown in Figure 2.

TABLE 3.-Heat treatment and average mechanical properties of the forgings cut in the lathe tests

CARBON STEEL S. A. E. $1040^{1}$

\begin{tabular}{|c|c|c|c|c|c|c|c|c|c|c|c|c|c|}
\hline \multirow{3}{*}{$\begin{array}{l}\text { Forg- } \\
\text { ing } \\
\text { No. }\end{array}$} & \multicolumn{4}{|c|}{ Heat treatment } & \multirow{3}{*}{$\begin{array}{l}\text { Pro- } \\
\text { por- } \\
\text { tional } \\
\text { limit }\end{array}$} & \multirow{3}{*}{$\begin{array}{l}\text { Yield } \\
\text { point }\end{array}$} & \multirow{3}{*}{$\begin{array}{l}\text { Tensile } \\
\text { strength }\end{array}$} & \multirow{3}{*}{$\begin{array}{l}\text { Elon- } \\
\text { gation } \\
\text { in } 2 \\
\text { inches }\end{array}$} & \multirow{3}{*}{$\begin{array}{l}\text { Re- } \\
\text { duc- } \\
\text { tion } \\
\text { of area }\end{array}$} & \multirow{2}{*}{\multicolumn{2}{|c|}{$\begin{array}{l}\text { Rockwell } \\
\text { hardness }\end{array}$}} & \multirow{3}{*}{$\begin{array}{l}\text { Shore } \\
\text { hard- } \\
\text { ness } \\
\text { No. }\end{array}$} & \multirow{3}{*}{$\begin{array}{l}\text { Brinell } \\
\text { hard- } \\
\text { ness } \\
\text { No. }\end{array}$} \\
\hline & \multicolumn{2}{|c|}{ Quenching s } & \multicolumn{2}{|c|}{ Tempering 4} & & & & & & & & & \\
\hline & $\begin{array}{l}\text { Tem- } \\
\text { pera- } \\
\text { ture }\end{array}$ & Medium & $\begin{array}{l}\text { Tem- } \\
\text { pera- } \\
\text { ture }\end{array}$ & Time & & & & & & B & C & & \\
\hline $\begin{array}{l}51 .-- \\
51 \mathrm{~A}- \\
51 \mathrm{~B}- \\
51 \mathrm{C}- \\
51 \mathrm{D}-\end{array}$ & $\begin{array}{l}{ }^{\circ} F \\
1,650 \\
1,650 \\
1,650 \\
1,650 \\
1,650\end{array}$ & 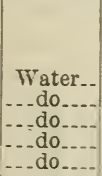 & $\begin{array}{r}{ }^{\circ} F \\
500 \\
800 \\
1,100 \\
1,300 \\
1,550\end{array}$ & $\begin{array}{c}\text { Hours } \\
6.5 \\
4 \\
4 \\
4 \\
4\end{array}$ & $\begin{array}{l}1,000 \\
\text { lbs.l } \\
\text { in.2 } \\
69.0 \\
64.0 \\
52.5 \\
47.0 \\
47.5\end{array}$ & $\begin{array}{l}1,000 \\
\text { lbs./ } \\
\text { in. } 2 \\
84.7 \\
76.0 \\
60.5 \\
54.0 \\
48.5\end{array}$ & $\begin{array}{c}1,000 \mathrm{lbs} . / \\
\text { in. } \\
123.6 \\
111.8 \\
95.7 \\
87.5 \\
78.0\end{array}$ & $\begin{array}{l}\text { Per } \\
\text { centt } \\
17.8 \\
20.5 \\
31.3 \\
31.5 \\
34.0\end{array}$ & $\begin{array}{l}\text { Per } \\
\text { cent } \\
55.5 \\
57.3 \\
64.2 \\
57.8 \\
53.5\end{array}$ & $\begin{array}{r}100.5 \\
96.0 \\
90.0 \\
87.0 \\
81.5\end{array}$ & $\begin{array}{r}20.6 \\
15.2 \\
9.5\end{array}$ & $\begin{array}{l}37 \\
36 \\
25 \\
27 \\
26\end{array}$ & $\begin{array}{l}241 \\
229 \\
184 \\
167 \\
149\end{array}$ \\
\hline
\end{tabular}

CHROMIUM-VANADIUM STEET, S. A. E. $6140^{1}$

\begin{tabular}{|c|c|c|c|c|c|c|c|c|c|c|c|c|c|}
\hline $\begin{array}{l}46 \ldots-. . \\
46 \mathrm{~A}-.- \\
46 \mathrm{~B}-.- \\
46 \mathrm{C}-. . \\
46 \mathrm{D}-. \\
46 \mathrm{E} . .\end{array}$ & $\begin{array}{l}1,650 \\
1,650 \\
1,650 \\
1,650 \\
1,650 \\
1,625\end{array}$ & 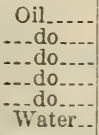 & $\begin{array}{r}500 \\
850 \\
1,100 \\
1,350 \\
1,550 \\
500\end{array}$ & $\begin{array}{r}3 \\
4 \\
4 \\
4 \\
4 \\
4\end{array}$ & $\begin{array}{l}98.5 \\
98.0 \\
87.0 \\
55.0 \\
42.5 \\
95.0\end{array}$ & $\begin{array}{r}114.0 \\
110.0 \\
90.0 \\
61.5 \\
49.8 \\
164.2\end{array}$ & $\begin{array}{r}145.4 \\
135.5 \\
114.3 \\
96.0 \\
90.4 \\
191.8\end{array}$ & $\begin{array}{l}15.0 \\
15.0 \\
20.5 \\
30.8 \\
29.5 \\
10.0\end{array}$ & $\begin{array}{l}55.8 \\
52.3 \\
60.8 \\
60.8 \\
53.5 \\
33.5\end{array}$ & $\begin{array}{r}106.5 \\
105.0 \\
100.0 \\
92.0 \\
39.0 \\
110.0\end{array}$ & $\begin{array}{l}30.1 \\
26.4 \\
21.2 \\
10.0 \\
37.3\end{array}$ & $\begin{array}{l}50 \\
41 \\
38 \\
31 \\
28 \\
55\end{array}$ & $\begin{array}{l}300 \\
290 \\
220 \\
192 \\
163 \\
372\end{array}$ \\
\hline
\end{tabular}

1 Reler to Table 2 for chemical composition.

2 Forgings Nos. 46, 47, 48, 49,50, and 51 were first annealed by heating at $1,400^{\circ}$ to $1,420^{\circ} \mathrm{F}$. and cooled in furnace to $1,100^{\circ}$ to $1,200^{\circ} \mathrm{F}$. and then cooled in air to room temperature. These forgings were anproximately 8 inches in diameter and hollow bored with holes 3 inches in diameter. Forging No. 46. had the same chemical composition and heat treatment as No. 46 except for the retempering treatment, $46 \mathrm{~B}$ has the same heat treatment as $46 \mathrm{~A}$ except for the retempering treatment, etc. Tbis procedure was followed in numbering and heat treating the forgings used in the tests with shallow cuts. The forgings at the time of the second quenching treatment were smaller in diameter than at the time of first quenching treatment. This change in size accounts for the higher tensile strengths and hardness obtalued in the latter case.

3 Forgings were heated with the furnace to the hardening temperutures in 3 to $1 \frac{1}{2}$ Lours, Leld at tew perutures from $1 \frac{1}{2}$ to $31 / 2$ hours and cooled as indicated.

Forgings Trere cooled in air from the tempering temperatures except those marked witb un asterisk (*), which were cooled slowly in the furnace. 
TABLE 3.- Heat treatment and average mechanical properties of the forgings cut in the lathe tests-Continued

CHROMIUM-MOLYBDENUM STEEL S. A. E. 41401

\begin{tabular}{|c|c|c|c|c|c|c|c|c|c|c|c|c|c|}
\hline \multirow{3}{*}{$\begin{array}{l}\text { Forg- } \\
\text { ing } \\
\text { No. }\end{array}$} & \multicolumn{4}{|c|}{ Heat treatment } & \multirow{3}{*}{$\begin{array}{l}\text { Pro- } \\
\text { por- } \\
\text { tional } \\
\text { limit }\end{array}$} & \multirow{3}{*}{$\begin{array}{l}\text { Yield } \\
\text { point }\end{array}$} & \multirow{3}{*}{$\begin{array}{l}\text { Tensile } \\
\text { strength }\end{array}$} & \multirow{3}{*}{$\begin{array}{l}\text { Elon- } \\
\text { gation } \\
\text { in } 2 \\
\text { inches }\end{array}$} & \multirow{3}{*}{$\begin{array}{c}\text { Re- } \\
\text { duc- } \\
\text { tion } \\
\text { of area }\end{array}$} & \multicolumn{2}{|c|}{$\begin{array}{l}\text { Rockwell } \\
\text { hardness }\end{array}$} & \multirow{3}{*}{$\begin{array}{l}\text { Shore } \\
\text { hard- } \\
\text { ness } \\
\text { No. }\end{array}$} & \multirow{3}{*}{$\begin{array}{c}\text { Brinel } \\
\text { hard- } \\
\text { ness } \\
\text { No. }\end{array}$} \\
\hline & \multicolumn{2}{|c|}{ Quenching } & \multicolumn{2}{|c|}{ Tempering } & & & & & & & & & \\
\hline & $\begin{array}{l}\text { Tem- } \\
\text { jers- } \\
\text { ture }\end{array}$ & Medium & $\begin{array}{l}\text { Tem- } \\
\text { pera- } \\
\text { ture }\end{array}$ & Time & & & & & & B & C & & \\
\hline $\begin{array}{l}47 \\
47 \mathrm{~A} \\
47 \mathrm{~B} \\
47 \mathrm{C} \\
4711 \\
47 \mathrm{E} \\
47 \mathrm{~F}\end{array}$ & $\begin{array}{l}{ }^{\circ} F . \\
1,625 \\
1,625 \\
1,625 \\
1,625 \\
1,625 \\
1,625 \\
1,625\end{array}$ & 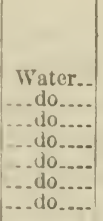 & $\begin{array}{r}{ }^{\circ} F \text {. } \\
700 \\
900 \\
1,100 \\
1,350 \\
1,550 \\
500 \\
750\end{array}$ & $\begin{array}{c}\text { Hours } \\
4 \\
4 \\
4 \\
4 \\
4 \\
4 \\
4\end{array}$ & $\begin{array}{c}1,000 \\
\text { lbs./ } \\
\text { in. } \\
79.0 \\
83.0 \\
70.0 \\
48.0 \\
35.0 \\
127.0 \\
110.0\end{array}$ & $\begin{array}{r}1,000 \\
\text { lbs./ } \\
\text { in.2. } \\
121.5 \\
96.2 \\
76.6 \\
54.3 \\
41.0 \\
165.8 \\
143.8\end{array}$ & $\begin{array}{r}1,000 \text { lbs./ } \\
\text { in.2 } \\
137.2 \\
120.7 \\
108.8 \\
93.7 \\
89.0 \\
210.9 \\
163.3\end{array}$ & $\begin{array}{l}\text { Per } \\
\text { cent } \\
18.0 \\
18.5 \\
26.5 \\
30.0 \\
28.0 \\
6.5 \\
13.8\end{array}$ & $\begin{array}{l}\text { Per } \\
\text { cent } \\
59.4 \\
59.5 \\
63.1 \\
60.7 \\
43.7 \\
31.8 \\
47.8\end{array}$ & $\begin{array}{r}104.5 \\
102.0 \\
95.5 \\
90.0 \\
87.5 \\
112.5 \\
108.5\end{array}$ & $\begin{array}{r}27.8 \\
23.9 \\
15.2 \\
7.9 \\
42.5 \\
33.8\end{array}$ & $\begin{array}{l}41 \\
38 \\
31 \\
30 \\
26 \\
59 \\
53\end{array}$ & $\begin{array}{l}287 \\
255 \\
204 \\
179 \\
166 \\
388 \\
321\end{array}$ \\
\hline
\end{tabular}

NICKEL-CHROMIUM STEEL S. A. E. 34351

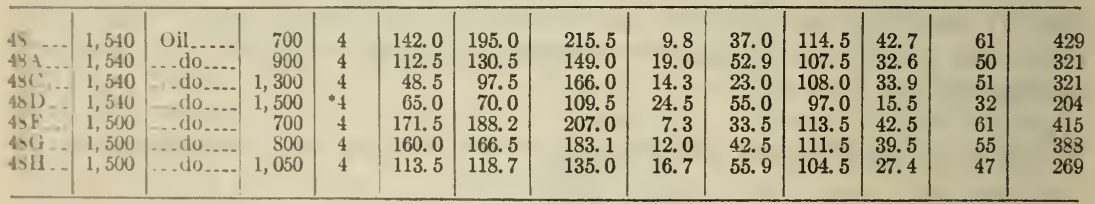

NICKEL-CHROMIUM STEEL S. A. E. $3140^{1}$

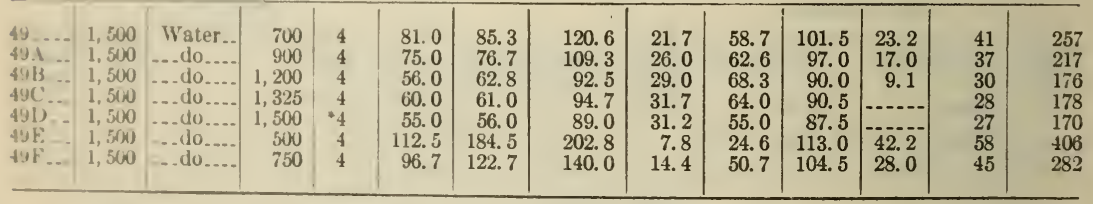

NICKEL STEEL S. A. E. $2340^{1}$

\begin{tabular}{|c|c|c|c|c|c|c|c|c|c|c|c|c|c|}
\hline 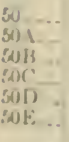 & $\begin{array}{l}1,525 \\
1,525 \\
1,525 \\
1,525 \\
1,525 \\
1,500\end{array}$ & 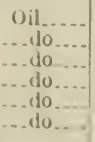 & $\begin{array}{r}500 \\
800 \\
1,100 \\
1,325 \\
1,500 \\
500\end{array}$ & $\begin{array}{r}3 \\
4 \\
4 \\
4 \\
4 \\
4\end{array}$ & $\begin{array}{r}71.5 \\
89.5 \\
72.0 \\
49.0 \\
61.7 \\
106.0\end{array}$ & $\begin{array}{r}125.5 \\
94.0 \\
78.3 \\
66.8 \\
63.5 \\
136.1\end{array}$ & $\begin{array}{l}150.0 \\
121.5 \\
105.8 \\
116.0 \\
101.6 \\
178.8\end{array}$ & $\begin{array}{r}13.2 \\
22.0 \\
27.3 \\
22.5 \\
24.8 \\
6.5\end{array}$ & $\begin{array}{l}45.3 \\
56.4 \\
64.8 \\
42.7 \\
44.0 \\
28.8\end{array}$ & $\begin{array}{r}106.5 \\
101.5 \\
97.5 \\
98.0 \\
93.5 \\
110.0\end{array}$ & \begin{tabular}{l|l|}
30.7 & \\
24.7 & \\
18.5 \\
18.3 \\
12.9 \\
36.5
\end{tabular} & $\begin{array}{l}42 \\
39 \\
34 \\
34 \\
28 \\
52\end{array}$ & $\begin{array}{l}302 \\
255 \\
215 \\
226 \\
193 \\
363\end{array}$ \\
\hline
\end{tabular}

1 Refer to Table 2 for chemical composition.

\section{RESULTS OF CUTTING TESTS}

The machining properties of a steel forging are recognized as being affected by (1) its chemical composition, (2) heat treatment after working, and (3) quality of the metal cut. The term "quality" refers to those details of composition and constitution not defined by ordinary chemical analysis or by a statement of the heat treatment, but which, nevertheless, may play a part in making a metal well suited for a particular service. So-called high quality for one service may be inferior for a different service; the term is general in nature as used in this report. It simply implies recognition of the fact thut there are other factors beside chemical composition and heat treatment which may contribute to the final properties of the metal. 
In the following experiments most attention was given to the first variable, chemical composition. However, the data throw some light upon the second variable, heat treatment; the quality of the metal was assumed to be practically constant in so far as each type composition
was concerned.

As is shown in Table 2, most of the forgings selected were alloy steels which contained approximately 0.4 per cent carbon. A plain carbon steel of similar carbon content was included so that comparisons could be made of the effects on tool performance or machinability of chromium, molybdenum, vanadium, and nickei, either alone or in combination.

The alloy steels were supplied through the courtesy of the Central Alloy Steel Corporation, now the Central Alloy Division of the Republic Steel Corporation, Massillon, Ohio. The plain carbon steel forging was contributed by the Illinois Steel Co., Chicago, Ill.

It is not practicable to select a single cutting speed with a fixed feed and depth of cut for tool testing on steel forgings with tensile strengths varying over the wide range of 75,000 to $220,000 \mathrm{lbs} / \mathrm{in}^{2}{ }^{2}$ For example, if a cutting speed was selected to give a tool life of 10 to 30 minutes on a forging heat treated to give a tensile strength of $90,000 \mathrm{lbs} . / \mathrm{in}^{2}{ }^{2}$ and then an attempt was made to use the same cutting condition on a forging of similar composition, but with a tensile strength of $200,000 \mathrm{lbs}$./in. ${ }^{2}$, the result would be immediate tool failure. Similarly, if the cutting speed was selected to give the desired tool life on the forging with high strength, then the tool life on the forging of lower strength would be extremely long. As already shown, the relation between the cutting speed and tool life of high-speed steel tools, under fixed feed and depth of cut can be closely represented by an empirical equation, and this fact was used in selecting the speeds at which the tool tests were made.

The comparisons of the different steels cut may conveniently be made on the basis of equal tensile strengths and the Taylor speed, such a comparison being taken as a measure of machinability.

TABLE 4.-Summary of lathe tests on different steels at 0.0115 inch per revolution feed and 0.010 inch depth of cut. ${ }^{1}$

CARBON STEEL S. A. E. 1040

\begin{tabular}{|c|c|c|c|c|c|c|c|c|c|c|c|}
\hline $\begin{array}{l}\text { Forg- } \\
\text { ing } \\
\text { No. }\end{array}$ & $\begin{array}{l}\text { Tensile } \\
\text { strength }\end{array}$ & $\begin{array}{l}\text { Cutting } \\
\text { speed }\end{array}$ & $\begin{array}{l}\text { Num- } \\
\text { ber of } \\
\text { tests } \\
\text { made }\end{array}$ & $\begin{array}{l}\text { A verage } \\
\text { tool } \\
\text { life }\end{array}$ & $\begin{array}{c}\text { Taylor }{ }^{2} \\
\text { speed }\end{array}$ & $\begin{array}{l}\text { Forg- } \\
\text { ing } \\
\text { No. }\end{array}$ & $\begin{array}{l}\text { Tensile } \\
\text { strength }\end{array}$ & $\begin{array}{l}\text { Cutting } \\
\text { speed }\end{array}$ & $\mid \begin{array}{c}\text { Num- } \\
\text { ber of } \\
\text { tests } \\
\text { made }\end{array}$ & $\begin{array}{c}\text { A verage } \\
\text { tool } \\
\text { lifo }\end{array}$ & $\begin{array}{c}\text { Taylor } 2 \\
\text { speed }\end{array}$ \\
\hline A. & $\begin{array}{r}1,000 \text { lbs. } \\
\text { in.2. } \\
123.6 \\
111.8 \\
95.7\end{array}$ & $\begin{array}{r}\text { Ft./min } \\
180 \\
200 \\
250\end{array}$ & $\begin{array}{l}8 \\
8 \\
7\end{array}$ & \begin{tabular}{|r|} 
Minutes \\
10.5 \\
7.4 \\
17.1
\end{tabular} & $\begin{array}{r}\text { F.. } / \mathrm{min} . \\
169 \\
181 \\
246\end{array}$ & $\begin{array}{l}\text { 51C... } \\
\text { 51D... }\end{array}$ & \begin{tabular}{|} 
1,000 lbs. \\
in.2. \\
87.5 \\
78.0
\end{tabular} & $\begin{array}{r}\text { Ft. } / \min . \\
310 \\
325\end{array}$ & $\begin{array}{l}8 \\
8\end{array}$ & $\begin{array}{r}\text { Minutes } \\
19.9 \\
12.0\end{array}$ & $\begin{array}{r}\text { Ft. } / \min . \\
310 \\
310\end{array}$ \\
\hline
\end{tabular}

CHROMIUM-VANADIUM STEEL S. A. E. 6140

\begin{tabular}{|c|c|c|c|c|c|c|c|c|c|c|c|}
\hline $\begin{array}{l}46 \ldots \\
46 \mathrm{~A}-\ldots- \\
46 \mathrm{~B}\end{array}$ & $\begin{array}{l}145.4 \\
135.5 \\
114.3\end{array}$ & $\begin{array}{l}200 \\
230 \\
280\end{array}$ & $\begin{array}{l}8 \\
8 \\
7\end{array}$ & $\begin{array}{l}13.4 \\
14.8 \\
14.1\end{array}$ & $\begin{array}{l}192 \\
223 \\
270\end{array}$ & $\begin{array}{l}46 \mathrm{C} \ldots- \\
46 \mathrm{~T} .-- \\
46 \mathrm{E} .--\end{array}$ & $\begin{array}{r}96.0 \\
90.4 \\
181.8\end{array}$ & $\begin{array}{l}290 \\
315 \\
120\end{array}$ & $\begin{array}{l}8 \\
8 \\
7\end{array}$ & $\begin{array}{l}32.3 \\
18.5 \\
26.0\end{array}$ & $\begin{array}{l}304 \\
313 \\
123\end{array}$ \\
\hline
\end{tabular}

1 The lathe tests were made dry with high-speed tool steel numbered AA. Composition and heat treatment of tools are given in Table 1 . Selected size and form of tool shown in Figure $1(B)$.

2 Computed from the average tool life by means of equation (1) of the text with $n=3 / 10$ or obtained from Figure 2. 
TABLE 4.-Summary of lathe tests on different steels at 0.0115 inch per revolution feed and 0.010 inch depth of cut-Continued

CHROMIUM-MOLYBDENUM STEEL S. A. E. 4140

\begin{tabular}{|c|c|c|c|c|c|c|c|c|c|c|c|}
\hline $\begin{array}{l}\text { Forg. } \\
\text { ing } \\
\text { Nio. }\end{array}$ & $\begin{array}{l}\text { Tensile } \\
\text { strength }\end{array}$ & $\begin{array}{l}\text { Cutting } \\
\text { speed }\end{array}$ & $\begin{array}{l}\text { Num- } \\
\text { ber of } \\
\text { tests } \\
\text { made }\end{array}$ & $\begin{array}{c}\text { A verage } \\
\text { tool } \\
\text { life }\end{array}$ & $\begin{array}{l}\text { Taylor } \\
\text { speed }\end{array}$ & $\begin{array}{l}\text { Forg- } \\
\text { ing } \\
\text { No. }\end{array}$ & $\begin{array}{l}\text { Tensile } \\
\text { strength }\end{array}$ & $\begin{array}{l}\text { Cutting } \\
\text { speed }\end{array}$ & $\begin{array}{l}\text { Num- } \\
\text { ber of } \\
\text { tests } \\
\text { made }\end{array}$ & $\begin{array}{c}\text { Average } \\
\text { tool } \\
\text { life }\end{array}$ & $\begin{array}{l}\text { Taylor } \\
\text { speed }\end{array}$ \\
\hline $\begin{array}{l}47 . \ldots . . \\
47 \AA . . . \\
4 i B . . \\
4 . C . . .\end{array}$ & $\begin{array}{c}1,00016 s . \\
\text { in. } \\
137.2 \\
120.7 \\
104.5 \\
93.7\end{array}$ & $\begin{array}{r}\text { Ft./min. } \\
180 \\
230 \\
320 \\
310\end{array}$ & $\begin{array}{l}8 \\
j \\
8 \\
8\end{array}$ & $\begin{array}{r}\text { Minutes } \\
41.2 \\
24.1 \\
11.2 \\
26.0\end{array}$ & $\begin{array}{r}\text { Ft./min. } \\
194 \\
234 \\
302 \\
318\end{array}$ & $\begin{array}{l}47 \mathrm{D} \ldots \\
47 \mathrm{E} \ldots \\
47 \mathrm{E} .-\end{array}$ & $\begin{array}{c}\text { 1,000 lbs. } \\
\text { in. } \\
89.0 \\
210.9 \\
163.3\end{array}$ & $\begin{array}{r}\text { Ft./min. } \\
340 \\
60 \\
150\end{array}$ & $\begin{array}{l}8 \\
8 \\
8\end{array}$ & $\begin{array}{r}\text { Minutes } \\
24.4 \\
19.1 \\
8.1\end{array}$ & $\begin{array}{r}\text { Ft./min. } \\
347 \\
60 \\
137\end{array}$ \\
\hline
\end{tabular}

NICKEL-CHROMIUM STEEL S. A. E. 3435

\begin{tabular}{|c|c|c|c|c|c|c|c|c|c|c|c|}
\hline $\begin{array}{l}4 . \ldots . . . \\
44 \mathrm{~A} . . . \\
45 \mathrm{D} \ldots\end{array}$ & $\begin{array}{l}215.5 \\
144.0 \\
160.0 \\
10 \% .5\end{array}$ & $\begin{array}{l}110 \\
200 \\
165 \\
215\end{array}$ & $\begin{array}{l}8 \\
8 \\
7 \\
8\end{array}$ & $\begin{array}{l}5.5 \\
2.3 \\
7.8 \\
8.7\end{array}$ & $\begin{array}{r}97 \\
161 \\
150 \\
198\end{array}$ & $\begin{array}{l}48 \mathrm{D} \ldots . . \\
48 \mathrm{~F} \ldots \\
48 \mathrm{G} \ldots \\
48 \mathrm{H} \ldots\end{array}$ & $\begin{array}{l}108.8 \\
207.0 \\
183.1 \\
135.0\end{array}$ & $\begin{array}{l}230 \\
110 \\
140 \\
190\end{array}$ & $\begin{array}{r}4 \\
8 \\
10 \\
8\end{array}$ & $\begin{array}{r}5.0 \\
10.4 \\
8.8 \\
9.0\end{array}$ & $\begin{array}{l}200 \\
103 \\
129 \\
175\end{array}$ \\
\hline
\end{tabular}

NICKEL-CEROMIUM STEEL S. A. E. 3140

\begin{tabular}{|c|c|c|c|c|c|c|c|c|c|c|c|}
\hline $\begin{array}{l}48 \ldots . . . \\
49 \mathrm{~A} . . . \\
49 \mathrm{~B} . .\end{array}$ & $\begin{array}{r}120.6 \\
109.3 \\
y 2.5 \\
94.7\end{array}$ & $\begin{array}{l}200 \\
250 \\
320 \\
320\end{array}$ & $\begin{array}{r}7 \\
7 \\
10 \\
8\end{array}$ & $\begin{array}{l}20.2 \\
14.6 \\
21.9 \\
16.8\end{array}$ & $\begin{array}{l}200 \\
242 \\
323 \\
314\end{array}$ & $\begin{array}{l}49 \mathrm{D} \ldots- \\
49 \mathrm{E} . .- \\
49 \mathrm{E} \ldots-\end{array}$ & $\begin{array}{r}89.0 \\
202.8 \\
140.0\end{array}$ & $\begin{array}{l}330 \\
110 \\
180\end{array}$ & $\begin{array}{l}8 \\
8 \\
8\end{array}$ & $\begin{array}{l}19.6 \\
17.9 \\
16.4\end{array}$ & $\begin{array}{l}329 \\
109 \\
177\end{array}$ \\
\hline
\end{tabular}

NICKEL STEEL S. A. E. 2340

\begin{tabular}{|c|c|c|c|c|c|c|c|c|c|c|c|}
\hline 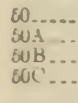 & $\begin{array}{l}150.0 \\
121.5 \\
105.8 \\
110.0\end{array}$ & $\begin{array}{l}180 \\
230 \\
300 \\
240\end{array}$ & $\begin{array}{l}8 \\
6 \\
8 \\
6\end{array}$ & $\begin{array}{l}19.4 \\
16.8 \\
13.8 \\
22.7\end{array}$ & $\begin{array}{l}179 \\
220 \\
290 \\
243\end{array}$ & $\begin{array}{l}50 \mathrm{D}-.- \\
50 \mathrm{D}-. . \\
50 \mathrm{E} . .-\end{array}$ & $\begin{array}{l}101.6 \\
101.6 \\
178.8\end{array}$ & $\begin{array}{l}280 \\
300 \\
100\end{array}$ & $\begin{array}{l}9 \\
7 \\
8\end{array}$ & $\begin{array}{r}12.1 \\
4.6 \\
17.0\end{array}$ & $\begin{array}{r}268 \\
259 \\
98\end{array}$ \\
\hline
\end{tabular}

The results of the tests with shallow cuts are given in Table 4 and summarized in Figure 3. These data show that if machinability with shallow cuts is measured by the cutting speed permitting the tool to last a definite time, then measurable and consistent differences are coserved in the machinability of the carbon and various alloy steels used in the experimental work. The fact, however, that some given steel permits a higher cutting speed than another steel for a selected tensile strength which is the same for both materials does not necessarily indicate that the two steels maintain the same relationship for mother tensile strength. This is illustrated in Figure 3 in that, for the conditions of cutting under consideration, the chromium-molybdemum steel (S. A. F. 4140) had the best machinability of the entire group of stecls at a tensile strength of 90,000 lbs./in. ${ }^{2}$ At a tensile strength of ubout $110,000 \mathrm{lbs}$./in." the chromium-vanadium steel (S. 1. F. $(140)$ had the best machinability of the group and retained this superiority up to a tensile strength in the neighborhood of 175,000 (1) $18(0,0001 \mathrm{~h}$. $/ 11$." At this point the tensile strength-Taylor speed curve for the chromium-vanadium steel crosses the curves for the nichel-chromium steels (S.A.E. 3140 and 3435) and above this range the lutter steels permit the higher cutting speeds.

As is also shown in Figure 3 , the slopes of the tensile strength'Taylor speed curves vary for the different steels. The curves for any twu compositions may cruss and so reverse the order of superiority. In some cases the slopes were not very different and there was an 
appreciable range in tensile strength at which, for all prastical purposes, the cutting speeds were the same.

The plain carbon steel (S. A. E. 1040), within the range of tensile strengths obtained by heat treatments, was the most difficult of the group to machine other than tho annealed nickel-chromium ster] (S. A. E. 3435). This would indicate that the special alloying elements-nickel, chromium, molybdenum, and vanadium-either alone or in combination, when added to the plain carbon stcel improve machinability from the standpoint of the cutting speed permitting a

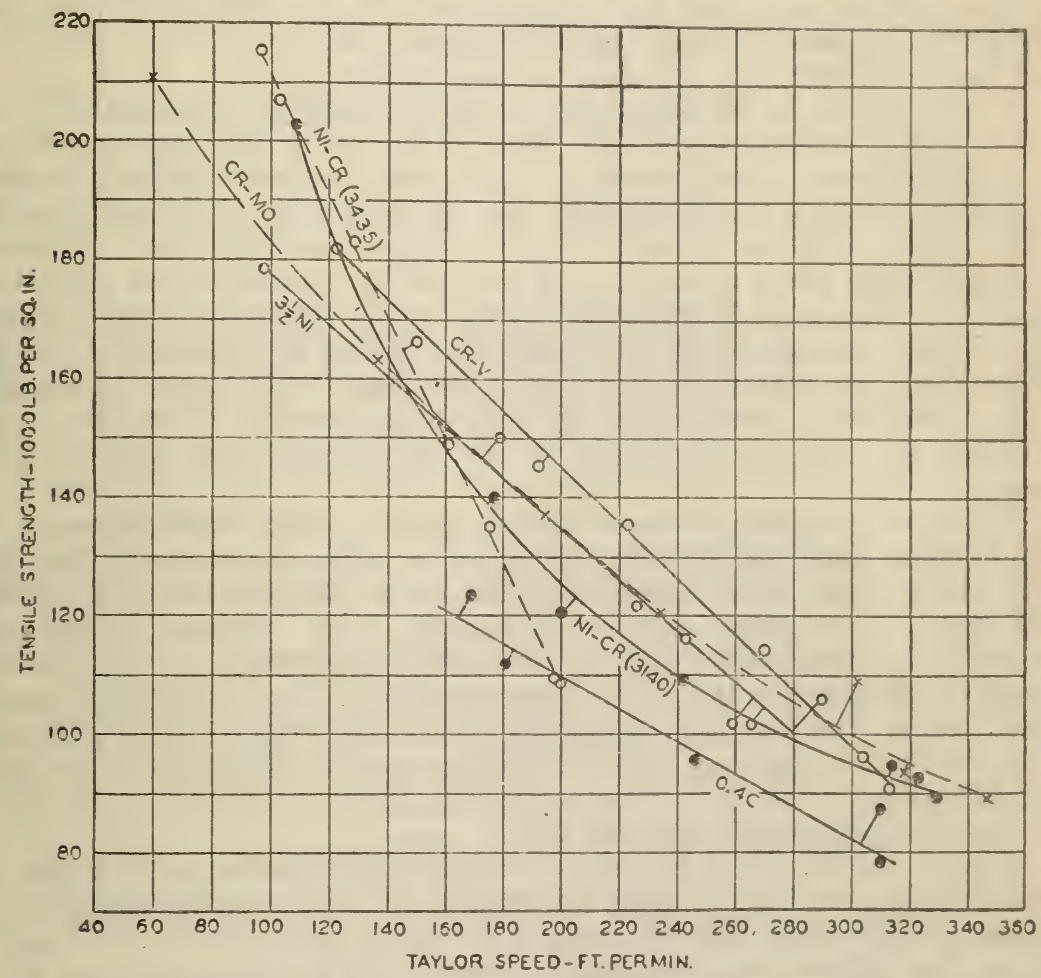

FIGURE 3.-Relation of the Taylor speed to the tensile strongth of the special steels cut in the lathe tests

For details of chemical composition and heat treatment of steel cut, refor in Tahles 2 and 3 . Ths composition and heat treatment of high-speed steel tools No. AA used in the tests are giren in Table 1. The lathe tests were made "dry" with the size and form of tool shown in Figure 1 (B), with 0.0115 inch per revolution feed and 0.010 inch depth of cut.

definite tool life with shallow cuts. However, the superiority in machinability of the alloy steels over the plain carbon type is not to be attributed solely to any single alloying element, but rather to the combined effects of the alloying elements present in any particular steel, considering carbon also as one of the alloying additions. This is evident from the fact that the same alloying elements which in one case increased the cutting speed, under other conditions, as, for example, when present in different relative proportions and requiring different heat treatments to give comparablo tensile strengths, may be without any marked effect on the cutting speed. 
This is illustrated by comparisons of the Taylor speeds of the nickelchromium steels with that of the carbon steel at a tensile strength of $110,000 \mathrm{lbs}$./in. ${ }^{2}$ The nickel-chromium steel containing 0.37 per cent carbon, 0.50 per cent chromium, and 1.26 per cent nickel had a Taylor speed of about 244 feet per minute, while the nickel-chromium steel containing 0.39 per cent carbon, 0.76 per cent chromium, and 2.96 per cent nickel showed the same Taylor speed as the plain 0.42 per cent carbon steel, namely, 198 feet per minute. Thus, at this particular tensile strength the addition of 0.5 per cent chromium and 1.26 per cent nickel increased the Taylor speed by 46 feet per minute, but higher proportions of nickel and chromium additions did not improve the Taylor speed over that of the plain carbon steel.

A comparison of the permissible cutting speeds or machinability of the nickel-chromium steels with those of the other alloy steels used in the experiments is of particular interest. At the higher tensile strengths superior machinability was secured with nickel-chromium alloy steels, but as already stated, certain combinations of these clements may cause a lowering of the cutting speeds of the annealed steels. As shown in Figure 3, the slope of the tensile strength-Taylor speed curve for the steel containing the higher proportions of nickel and chromium was such as to permit a very wide range of tensile values without a marked change in cutting speeds. This fact may possibly be used to advantage in the industrial application of this steel.

With the exception of the nickel-chromium steels, the differences in the Taylor speeds of the alloy steels were within a range of about 20 feet per minute when considering steels of $100,000 \mathrm{lbs}$./in. ${ }^{2}$ tensile strength. The range increased slightly with increase in tensile strength and reached a value of about 30 feet per minute when cutting steels of $170,000 \mathrm{lbs}$./in. ${ }^{2}$ The Taylor speed for the $3 \frac{1}{2}$ per cent nickel steel was on the low side throughout the entire range. Thus, the $3 \frac{1}{2}$ per cent nickel steel was more difficult to machine than the chromiumvanadium steel, and was either more difficult or as difficult to machine as the chromium-molybdenum steel.

The results shown in Figure 3 indicate that the effect of changes in chemical composition of steel forgings upon their cutting speeds was dependent upon the tensile strength at which comparisons were made. Since the highest tensile strengths considered in these tests are produced by quenching, with or without subsequent tempering, changes in chemical composition may act in opposite directions depending upon whether the steel cut is close to the fully hardened (martensitic) or the annealed (pearlitic) condition.

Elements which cause a lowering in the cutting speeds of the annealed steels may improve the cutting speeds when considering higher tensile strengths. Of the special elements that improved machinability of the different steels cut with shallow cuts, the most effective were the combinations of nickel and chromium or chromium and vanadium for the higher tensile strengths in the neighborhood of $180,000 \mathrm{lbs} / \mathrm{in} .{ }^{2}$ while the combination of chromium and molybdenum was the most effective in the lower range of about 90,000 lbs./in. ${ }^{2}$

The mechanical properties of the forgings, given in Table 3 , and the cutting test results given in Table 4 permit comparisons to be made other than on the tensile strength-Taylor speed basis as given in the 
text. The relative order of machinability of the different steel forgings when comparisons were made of the Brinell hardness numbers-Taylor speed agreed very closely with the order as given by the tensile strength-Taylor speed basis. This agreement might be expected. The order of machinability as given by the tensile strengthTaylor speed was changed when comparisons were made on the basis of the Rockwell hardness (B or C seale)-Taylor speed or Shore hardness-Taylor speed.

TABLE 5.-Effect of method of heat treatment on the cutting speeds of steel forgings having equal tensile strengths

COMPARISON OF DIFFERENT METHODS OF QUENCHING AND TEMPERING

\begin{tabular}{|c|c|c|c|c|}
\hline $\begin{array}{c}\text { Test } \\
\text { forging } \\
\text { No. }\end{array}$ & Type composition (in per cent) & Heat treatment & $\begin{array}{c}\text { Tensile } \\
\text { strength }\end{array}$ & $\begin{array}{l}\text { Taslor } \\
\text { speed }\end{array}$ \\
\hline 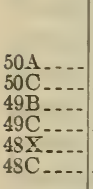 & $\begin{array}{l}0.36 \text { carbon, } 3.4 \text { nickel } \\
0.37 \text { carbon, } 0.5 \text { chromium, } 1.3 \text { nickel } \\
0.39 \text { carbon, } 0.8 \text { chromium, } 3.0 \text { nickel }\end{array}$ & 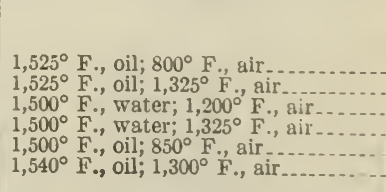 & $\begin{array}{r}1,000 \\
\text { lbs./in.2 } \\
121.5 \\
116.0 \\
92.5 \\
94.7 \\
1166.0 \\
166.0\end{array}$ & $\begin{array}{r}\text { ft. } / \min \\
226 \\
243 \\
323 \\
314 \\
2145 \\
150\end{array}$ \\
\hline
\end{tabular}

COMPARISON OF QUENCHED AND TEMPERED STEELS WITH ANNEALED STEELS

\begin{tabular}{|c|c|c|c|c|}
\hline $\begin{array}{l}51 \mathrm{C} \ldots \\
51 \mathrm{D} \ldots-. . \\
46 \mathrm{C} \ldots .-\end{array}$ & 0.42 carbon 0.39 carbon, 1.0 chromium, 0.17 vana- & $\begin{array}{l}1,650^{\circ} \mathrm{F} ., \text { water; } 1,300^{\circ} \mathrm{F} . \text {, air...... } \\
1,550^{\circ} \mathrm{F} ., \text { cooled slowly in furnace... } \\
1,650^{\circ} \mathrm{F} ., \text { oil; } 1,350^{\circ} \mathrm{F} ., \text { air.......... }\end{array}$ & $\begin{array}{l}87.5 \\
78.0 \\
96.0\end{array}$ & $\begin{array}{l}310 \\
310 \\
304\end{array}$ \\
\hline $\begin{array}{l}46 \mathrm{D} \\
47 \mathrm{C}\end{array}$ & $\begin{array}{l}0.39 \text { carbon, } 0.9 \text { chromium, } 0.17 \text { molyb- } \\
\text { denum. }\end{array}$ & $\begin{array}{l}1,550^{\circ} \mathrm{F} ., \text { cooled slowly in furnace... } \\
1,625^{\circ} \mathrm{F} ., \text { water; } 1,350^{\circ} \mathrm{F} . \text {, air..... }\end{array}$ & $\begin{array}{l}90.4 \\
93.7\end{array}$ & $\begin{array}{l}313 \\
318\end{array}$ \\
\hline $\begin{array}{l}47 \mathrm{D} \\
49 \mathrm{C} \ldots-\ldots- \\
49 \mathrm{D} \\
50 \mathrm{~B} \ldots-\ldots- \\
50 \mathrm{D} \ldots\end{array}$ & $\begin{array}{l}0.37 \text { carbon, } 0.5 \text { chromium, } 1.3 \text { nickel } \\
0.36 \text { carbon, } 3.4 \text { nickel }\end{array}$ & 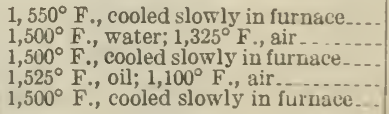 & $\begin{array}{r}89.0 \\
94.7 \\
89.0 \\
105.8 \\
101.6\end{array}$ & $\begin{array}{l}347 \\
314 \\
329 \\
290 \\
266\end{array}$ \\
\hline
\end{tabular}

1 Estimated from the known relation between tempering temperature and tensile strength.

2 Value obtained from tensile strength, Taylor speed curve of Figure 3.

Note. - The lathe tests were made dry with high-speed tool steel AA (Table 1), with a feed of 0.0115 inch per revolution and 0.010 inch depth of cut. Selected form and size of tool shown in Figure $1(B)$.

The heat treatments of the forgings, as given in Table 3 , show that the desired range of tensile properties were obtained by annealing, or by quenching, followed by tempering at different temperatures. The results also show that in some cases approximately equal tensile strengths were produced with a given forging by varying the methods of heat treatment.

The data given in Table 5 show that, in general, the cutting speeds were not appreciably affected by the method of heat treatment by which a given tensile strength was produced. The cutting speeds are slightly higher with the high tempering temperatures when comparisons are made of the different methods of quenching and tempering to produce approximately equal strengths. In two cases, namely, with the plain carbon steel (S. A. E. 1040) and $3 \frac{1}{2}$ per cent nickel steel (S. A. E. 2340) the better machinability was produced by the heat treatment consisting of quenching and subsequently tempering at a high temperature than with the annealing treatment used. 


\section{TESTS WITH COBALT HIGH-SPEED STEEL TOOLS}

High-speed steel tools containing considerable proportions of cobalt have been reported to give excellent performance when cutting hard metals and to have made possible the commercial machining of high manganese steel. In previous tests made at the National Bureau of Standards, ${ }^{11}$ it was found that cobalt improved the performance of high-speed steel lathe tools, with both shallow and roughing cuts,

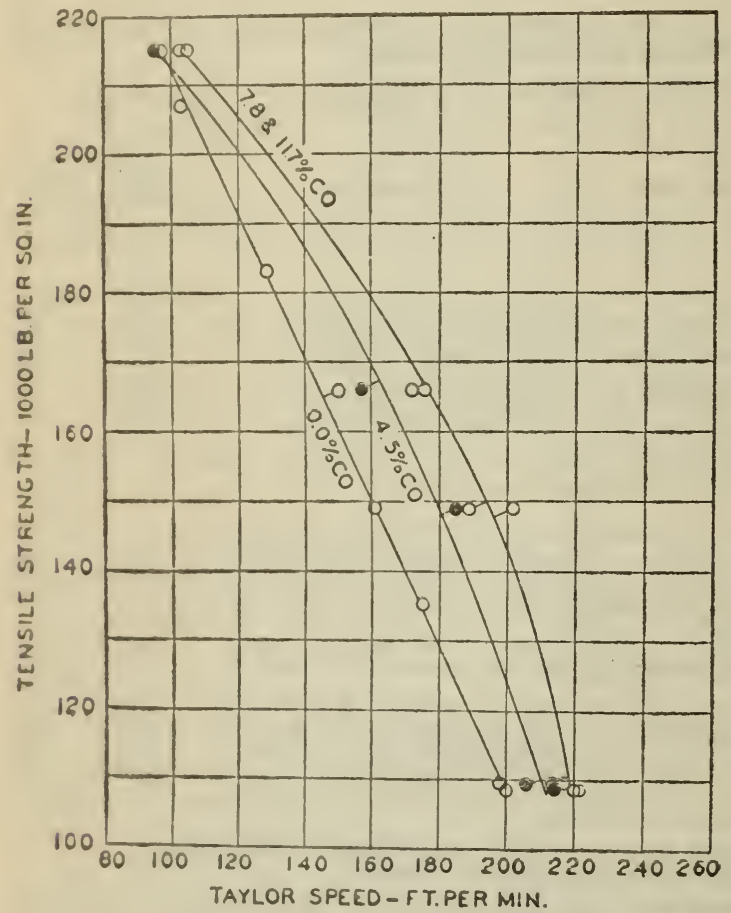

Figure 4.-Relation of the Taylor speed to the tensile strength of the nickel-chromium (S.A. E. 3435) steel cut with high-speed steel tools containing different proportions of cobalt

Chemical composition and heat treatment of the high-speed steel tuols are glveu in 'Table 1. Chemical composition, heat treatment, and tuechanical properties of the steel cut are given in Tables 2 and 3 . The lathe tests were made "dry" with the size and form of tool shown in Figure 1 (B), with fled of 0.0115 inch per revolution and 0.010 inch

but that the maximum benefits were obtained only with high-hardening temperatures.

The proportionate gain in machinability from the addition of cobalt to high-speed steel tools was somewhat greater with rough turning than with shallow cuts, butincrease in cobalt above about 5 per cent did not produce improvements commensurate with those resulting from additions of from 3.5 to 5 per cent together with high-hardening temperatures. These conclusions were made from experiments in cutting a 0.3 per cent carbon, $31 / 2$ per cent nickel steel forging heat treated to give a tensile strength of about 90,000 to 100,000 lbs./in. ${ }^{2}$. Since highspeed steel tools containing high proportions of cobalt are
d materials, the tests chumed to be especially adapted to cutting hard materials, the tests
with shallow euts were extended to include the cutting of nickel chromium steel forgings (S. A. E. 3435) having tensile strengths letween 100,000 and $220,000 \mathrm{lbs}$. $/ \mathrm{in} .^{2}$.

The results of the cutting test carried out in the present investigation with high-speed steel tools, with and without cobalt, are summarized in Figure 4. The data show that lathe-tool performance with shallow cuts was improved by the additions of cobalt (together with higher hardening temperatures) to the customary 18 per cent 


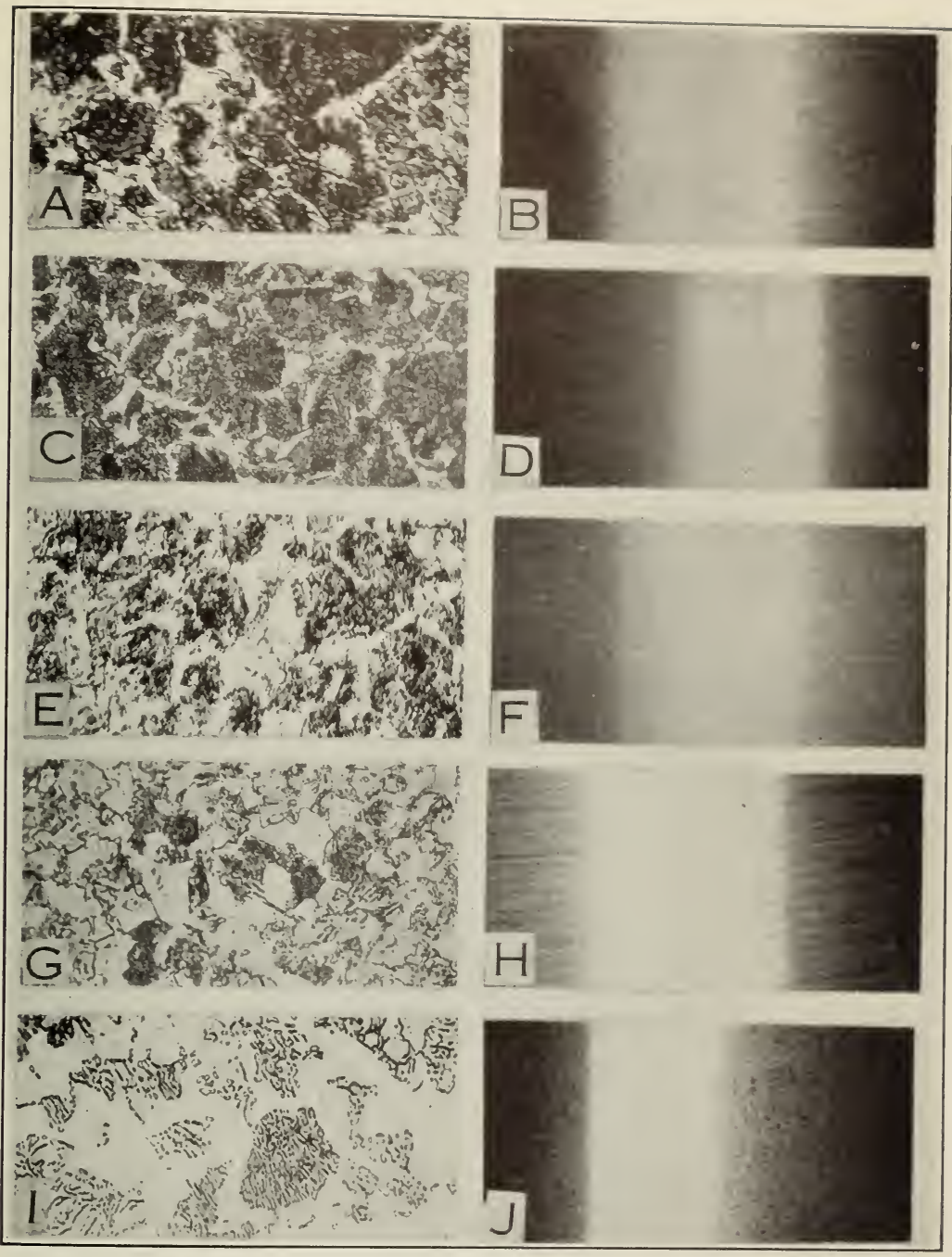

Figure 5.-Microstructures and surface finishes of plain carbon (S. A. E. $1040)$ steel cut in the lathe tests

For details of chemical composition and heat treatment of the forgings refer to Tables 2 and 3 . The lathe tests were made "dry" with high-speed steel tools (AA) of size and form shown in Figure 1 $(B)$, with 0.0115 inch per revolution feed and 0.010 inch depth of (nit.

\begin{tabular}{|c|c|c|c|c|c|}
\hline $\begin{array}{l}\text { Photomicrograph }(\times 500) \text { etched } \\
\text { with } 2 \text { per cent nitric acid in alcohol }\end{array}$ & $\begin{array}{c}\text { Surface } \\
\text { finish } \\
(\times 1)\end{array}$ & $\begin{array}{l}\text { Forging } \\
\text { No. }\end{array}$ & $\begin{array}{l}\text { Tensile } \\
\text { strength }\end{array}$ & $\begin{array}{l}\text { Cntting } \\
\text { speed }\end{array}$ & $\begin{array}{l}\text { Taylor } \\
\text { spleed }\end{array}$ \\
\hline $\begin{array}{l}\mathrm{A} \\
\mathrm{C} \\
\mathrm{E} \\
\mathrm{E} \\
\mathrm{G} \\
\mathrm{I}_{2}\end{array}$ & $\begin{array}{l}\text { B } \\
\text { I) } \\
\text { F } \\
\text { HI } \\
\text { J }\end{array}$ & $\begin{array}{l}51 \\
511 \\
5113 \\
510 \\
511\end{array}$ & $\begin{array}{c}\text { 1.000lbs./in.2 } \\
123.6 \\
111.8 \\
95.7 \\
\text { तi. } 5 \\
\text { is. } 0\end{array}$ & $\begin{array}{l}\text { Ft./min. } \\
140 \\
200 \\
250 \\
310 \\
325\end{array}$ & $\begin{array}{c}\text { Ft. } / \min . \\
16 ! \\
151 \\
216 \\
310 \\
310\end{array}$ \\
\hline
\end{tabular}




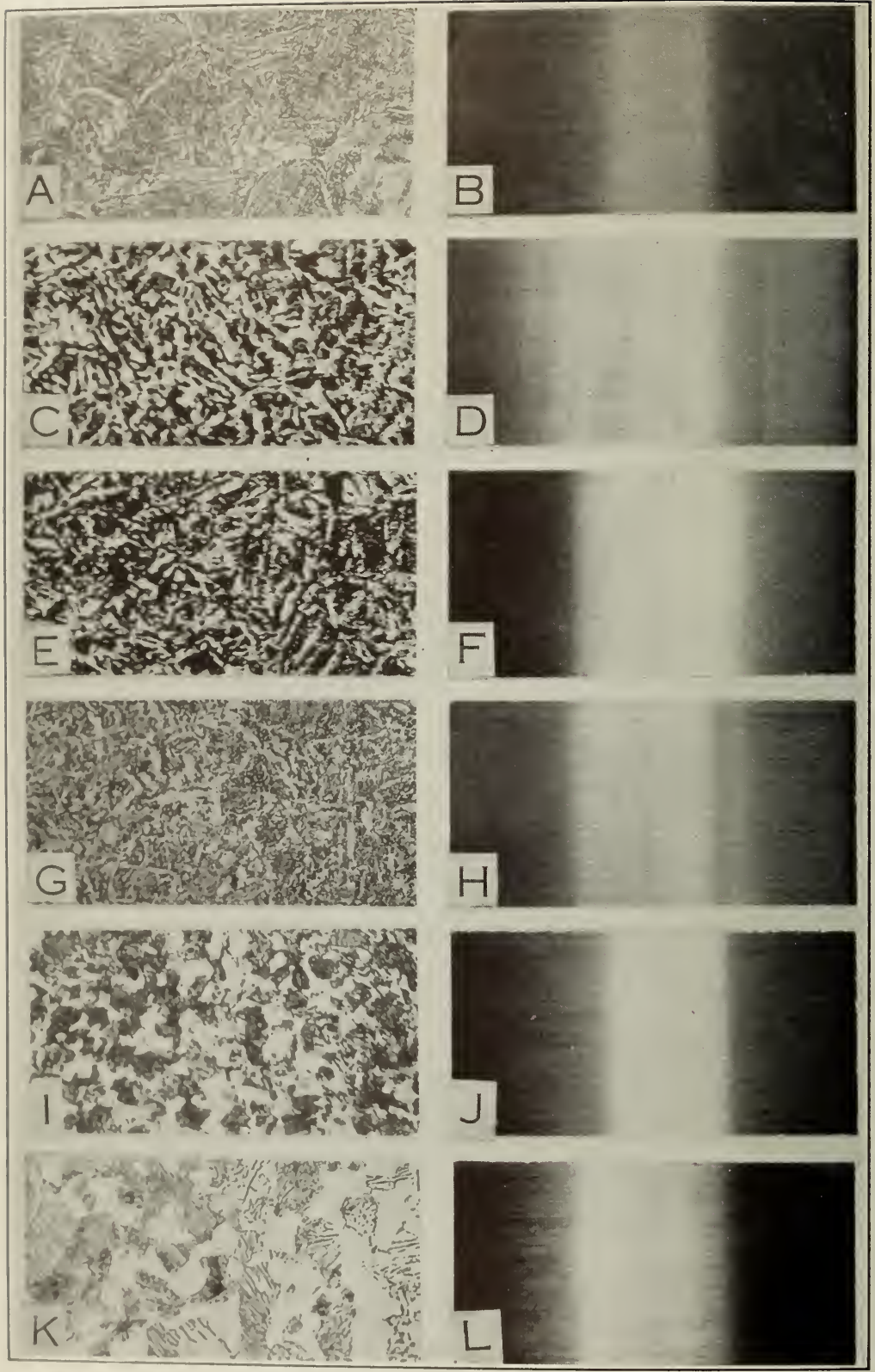

Fut UE, 6.-Microstructures and surface finishes of chromium-vanadium (S. A. F. 6140) steel cut in the lathe tests

For det al of limmical composition and heat treatment of the forgings refer to Tables 2 and 3. The Thin te tr were male "dry " with, high-speed steel tools (A A) of size and form shown in Figure 1 (b), w th 19.0115 inch trer revolution feed and 0.010 inch depth of cut.

\begin{tabular}{|c|c|c|c|c|c|}
\hline 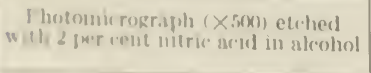 & $\begin{array}{c}\text { Surface } \\
\text { finish } \\
(\times 1)\end{array}$ & $\begin{array}{l}\text { Forging } \\
\text { No. }\end{array}$ & $\begin{array}{l}\text { Tensile } \\
\text { strength }\end{array}$ & $\begin{array}{l}\text { Cutting } \\
\text { speed }\end{array}$ & $\begin{array}{l}\text { Taylor } \\
\text { speed }\end{array}$ \\
\hline 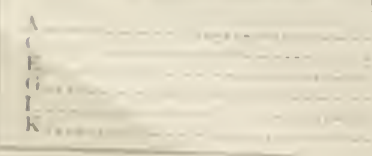 & $\begin{array}{l}\text { I3 } \\
\text { I) } \\
\text { F } \\
\text { II } \\
\text { J } \\
\text { I, }\end{array}$ & $\begin{array}{l}46 \mathrm{E} \\
46 \\
46 \mathrm{~A} \\
46 \mathrm{~B} \\
46 \mathrm{C} \\
46 \mathrm{D}\end{array}$ & \begin{tabular}{|c|} 
1,00olbs./in.$^{2}$ \\
181.8 \\
145.4 \\
135.5 \\
114.3 \\
96.0 \\
90.4
\end{tabular} & $\begin{array}{c}\text { Ft./min. } \\
70 \\
200 \\
230 \\
250 \\
290 \\
315\end{array}$ & $\begin{array}{l}\text { Ft./min. } \\
123 \\
192 \\
223 \\
270 \\
304 \\
313\end{array}$ \\
\hline
\end{tabular}


tungsten type of high-speed steel. Maximum gain in performance was obtained when cutting the forgings with tensile strengths up to about $170,000 \mathrm{lbs} . /$ in. $^{2}$; above this strength the gain in performance by increasing the cobalt additions was not so marked. Confirmation was also obtained of the previous test results, namely, that the increase in cobalt above about 5 per cent did not produco improvements of the same order as those resulting from 3.5 to 5 per cent, together with high-hardening temperatures.

\section{MICROSTRUCTURES AND SURFACE FIITISHES}

Although most attention was given to tool life with shallow culti in studying the machinability of the different forgings cut in the lathe tests, it was also recognized that the structure and appearance. of the surface finish are important factors in finishing cuts and possibly may be considered as factors in any index to machinability.

Photographs were made of representative surface conditions whilo cutting with sharp tools for each series of cutting tests, and these photographs were later compared with the appearances as recorded by the observer during the machining operations. Thero was a fair agreement between the classification by the two mothods. It was found that the photographs were a convenient but not an entirely satisfactory basis for making comparisons. All photographs were "natural size," and no attempts were made to show the surface conditions at greater magnifications.

The samples for microscopic examination were etched in 2 per cent nitric acid in alcohol and all micrographs are given at 500 magnifications. The microstructure shown in Figure $5(G)$ corresponded to the best surface finish (shown at fig. $5(H)$ ) of the entire series of plain carbon steels (S. A. E. 1040) used in the experiments with shallow cuts. The resulting surface was fairly smooth and polished, with some tool marks; and while not so good as some of the surfaces produced on the different alloy steel forgings, it may be classed as satisfactory. The finishes as shown in Figure $5(B),(D),(F)$, and $(J)$ were dull, badly torn, with small bright particles embedded in the surface. None of the latter conditions were considered sati-ifactory so far as the appearance of the finish was concerned.

An interesting feature is shown by comparing the heat treatments, microstructures, tensile properties, finishes, and machinability of forgings $51 \mathrm{C}$ and $51 \mathrm{D}$. Forging $51 \mathrm{C}$, quenched from $1,650^{\circ} \dot{\mathrm{H}}$. in water and subsequently tempered at $1,300^{\circ} \mathrm{F}$. and cooled in air shows some spheroidization or agglomeration of the cementite (fig. $5(G))$, satisfactory surface finish, 87,500 lbs./in. ${ }^{2}$ tensile strength, and a Taylor speed of 310 feet per minute. Forging 51D, quenched from $1,650^{\circ} \mathrm{F}$. in water and subsequently annealed at $1,550^{\circ} \mathrm{F}$, slowly cooled in furnace, shows distinctly lamellar pearlite (fig. $5(I)$ ), $81,500 \mathrm{lb}$. in. $^{2}$ tensile strength, and a Taylor speed of 310 feet per minute. Forging $51 \mathrm{C}$, therefore, showed the best machinability both from the viewpoint of the cutting speed and appearance of the surface finish, provided comparisons of cutting speeds were made on the basis of equal tensile strengths.

For the cutting conditions investigated, the chromium-vanadium, chromium-molybdenum, nickel-chromium (3135), and $3 \frac{1}{2}$ per cent nickel-steel forgings were considered as producing surfaces of ap- 
proximately equivalent smoothness, and were slightly superior to the nickel-chromium steel (3435). However, there were no very marked differences observed in the appearance of the surfaces of the forgings of the different alloy compositions, and all may be classified as satisfactory. Each type of alloy steel showed surfaces that were considered much superior to that of the plain carbon steel.

The characteristic finish of the different alloy steels may be described as smooth, medium to highly polished, with but little tearing and showing some tool marks.

The microstructures and surface finishes of all the alloy steel forgings with the varying heat treatments were examined, but no correlation between structure, surface finish, and machinability appeared possible. Figure 6 showing the microstructures and surface finishes of the chromium-vanadium forgings is representative of the results obtained with the ailoy groups.

Vanick and Wickenden ${ }^{12}$ have shown in some lathe tests of plain and alloy low-carbon steels of the carburizing type that for each steel and its particular heat treatment there was a critical range of rolume removal rates within which a rough finish was obtained. $\mathrm{By}$ aroiding this critical range, smoothly finished surfaces could be obtained. It was found that cutting conditions leaving a rough surface could be changed to give a smooth finish by (1) either lowering, or preferably increasing the cutting speed until it is outside the critical range; (2) maintaining the speed, but changing the cut or feed; (3) sharpening the cutting angle of the tool and maintaining speed and shape of chip; (4) changing the hardness of the steel being cut; usually increasing it in order that a good finish is produced at an casily obtainable speed.

Rapatz ${ }^{13}$ in a study of the surface conditions of plain-carbon and nickel-chromium steels of different strengths and hardness in some lathe turning tests found that higher tensile strengths, higher speeds, and greater depths of cut favored the production of smooth surfaces, assuming turning is performed with perfect tools. Yield point, elongation, and reduction of area were less important for obtaining smooth surfaces.

Although some of the surface finishes of the alloy steel forgings used in the present investigation were more highly polished and came nearer to being smooth than others, the differences observed in the general characteristics of the finishes were not large. The order in which the forgings were arranged in regard to the surface appearance under the conditions of test used, possibly might be different if the cutting conditions were changed.

\section{SUMMARY AND CONCLUSIONS}

1. The tests described in this report were made primarily as a study of high-speed steel tool performance with shallow cuts as affected by variations in the chemical composition and heat treatment of the steels cut. The study also included consideration of the surface finish of the various steel forgings, the microstructures of the metals cuit, and also of the tool performance as affected by addi-

Trans. A m. Finick and T. H. Wickenden, Smnoth Finish Machining of Low-Carbon Plain and Alloy Steels, Trans. Am. Foc. Steel Treat., 11, pp. 5.51; 1927.

Fisenhutenwesen, The Surface Conditions of Materials in Machining, Especially Turning, Archiv. fur 
tions of from 3.5 to 11.7 per cent cobalt to the 18 per cent tungsten type of high-speed steel.

2. The method used for testing lathe tools with shallow cuts was based upon the fact that when two tools are set at equal depths in one tool holder the second, or indicating tool, will not cut so long as the leading, or test tool, shows no wear. With this method of test, the indicating tool began to cut when the wear on the test tool was from 0.001 to 0.002 inch and this was considered as the point of failure of the test tool. In most cases, it was found that the wear of 0.001 to 0.002 inch coincided with a complete brenkduwn of the tool comparable to that found with heavy cuts in rough turning.

3. The lathe-cutting tests were made dry with high-speed steel tools of a selected size, form, composition, and heat treatment, with a fixed feed of 0.0115 inch per revolution, 0.010 inch depth of cut, and variable cutting speeds, depending upon the properties of the material cut.

4. The metals cut included a plain carbon and various alloy steel forgings heat treated to give tensile strengths between 75,000 and $220,000 \mathrm{lbs}$./in. ${ }^{2}$

5. The measure of machinability was the cutting speed permitting a definite tool life.

6. Measurable and consistent differences were observed in the machinability of the carbon and alloy steels used. 'The fact, however, that some given steel permits a higher cutting speed than another steel for some tensile strength which is the same for both materials does not necessarily indicate that the two stecls maintain the same relationship for another tensile strength.

7. The 0.4 per cent plain carbon steel, within the range of tensilo strengths obtained by heat treatments, was the most difficult to machine with shallow cuts other than an annealed nickel-chromium steel. The surface finishes of the carbon steel forgings were also inferior to those produced on the alloy steel forgings. However, with a particular heat treatment of the plain carbon stecl, showing a micrnstructure of partially spheroidized or agglomerated cementite, a medium smooth and satisfactory finish was obtained with the cutting condition used. This same heat treatment also resulted in the best machinability of the group of carbon steels when comparisons were made on the basis of the cutting speeds at equal tensile strengths.

8. The superiority in cutting speeds of the alloy steels over the plain carbon type is not to be attributed solely to any single alloying element, but rather to the combined effects of the alloying elements present in any particular steel, considering carbon also as one of the alloying additions.

9. The results of the present tests with shallow cuts compared with previously reported tests with roughing cuts ${ }^{14}$ show that the effect of changes in chemical composition of stecl forgings upon their cutting speeds is dependent not only upon the tensile strength at which comparisons are made, but also upon the conditions of cutting. That is, steel forgings that show superior machinability with shallow cuts at some tensile strength do not necessarily show a similar superiority with roughing cuts.

10. Of the special elements that improved machinability of the different steels cut with shallow cuts, the most effective were the

14 See footnote 4, p. 978. 
combinations of nickel and chromium or chromium and vanadium for the higher tensile strenghths, in the neighborhood of 180,000 lbs./in. ${ }^{2}$ and chromium and molybdenum in the lower range of tensile strengths, about $90,000 \mathrm{lbs} . /$ in. $^{2}$

11. In general, the cutting speeds were not appreciably affected by the method of heat treatment by which a given tensile strength was produced. The cutting speeds were slightly higher with the higher tempering temperature when comparisons were made of different methods of quenching and tempering to produce approximately equal tensile strengths. In two steels, namely, with the plain carbon steel and $3 y_{2}^{\prime}$ per cent nickel steel, the better machinability was produced by the heat treatment consisting of quenching and subsequently tempering at a high temperature than with the annealing treatments used.

12. Lathe-tool performance with shallow cuts was improved by the additions of cobalt (together with higher hardening temperatures) to the customary 18 per cent tungsten type of high-speed tool steel. The maximum gain in performance was shown when cutting the forgings with tensile strengths up to about $170,000 \mathrm{lbs}$./in. ${ }^{2}$, but above this strength the gain in performance was not so marked.

13. Confirmation was also obtained of previous test results, namely, that the increase in cobalt above about 5 per cent did not produce improvements in tool performance of the same order as those resulting from $3 \frac{1}{2}$ to 5 per cent and higher hardening temperatures.

14. The differences observed in the surface finish of the different types of alloy' steel forgings were not large and all were considered as being satisfactory and of about equivalent smoothness.

15. A correlation of the cutting speeds, tool life, surface finish, etc., shows that, with the test method used, the machinability of the carbon and different alloy steel forgings used in the experiments may be properly determined or measured by the cutting speed permitting the tool to last a definite time.

\section{ACKNOWLEDGMENTS}

The writer acknowledges the assistance of E. C. Smith, chief metallurgical engineer, formerly of the Central Alloy Steel Corporation, Massillon, Ohio, for supplying the alloy steel forgings; and to S. Epstein, metallurgist, formerly of the Illinois Steel Co., South Chicago, Ill., for supplying the carbon steel forging used in the cutting tests.

Grateful acknowledgment is also due to W. V. Magruder, laboratory aid, formerly of the National Bureau of Standards, for his yssistance in carrying out the many tests; to Louis Jordan, senior metallurgist, for his suggestions in the presentation of the test data; and to S. E. Sinclair, junior metallurgist, for the micrographs given in this report.

Washington, March 18, 1931. 